\title{
Evolução da autoconfiança e segurança de estudantes após aprendizagem utilizando manequins em Periodontia
}

\author{
Mariane Izabele Forte*; Paulo Henrique Chagas*; Gabriel Guidio Guarenghi**; João Paulo \\ Steffens $* * *$ \\ * Graduando em Odontologia, Universidade Federal do Paraná \\ ** Mestrando em Odontologia, Universidade Federal do Paraná \\ *** Professor, Departamento de Estomatologia, Universidade Federal \\ do Paraná
}

Recebido em 24/10/2018. Aprovado em 05/06/2019.

\begin{abstract}
RESUMO
A simulação no ensino em saúde é uma ferramenta que permite ao estudante uma aprendizagem em ambiente protegido e controlado, livre de intercorrências e com devolutiva instantânea da tarefa desempenhada. Na simulação, o docente pode intervir a qualquer momento, o que o auxilia na avaliação e instrução do estudante. O objetivo deste estudo foi avaliar a evolução da autoconfiança e segurança de estudantes após aprendizagem simulada utilizando manequim em um componente curricular de Periodontia e sua correlação com desempenho prático. Questionários utilizando escala visual analógica (EVA), escala numérica de 101 pontos (NRS-101) e escala verbal de 4 pontos (VRS4) foram aplicados aos estudantes da Disciplina de Periodontia I da Universidade Federal do Paraná no primeiro semestre de 2018, em três momentos: 1) antes do início das atividades práticas; 2) após a fase laboratorial; e 3) após a fase clínica. Os dados obtidos foram comparados entre si pelo tempo de aplicação e correlacionados com a nota prática final. Os estudantes demonstraram maior autoconfiança e segurança no final do que no início do período (Friedman; p<0,05). Essa evolução foi notada principalmente entre a primeira e segunda aplicações dos questionários, ou seja, após a fase laboratorial simulada em manequim. Houve correlação entre autoconfiança e segurança aferidas pela NRS-101 e o desempenho prático (Spearman; $r=0,3948$ e $r=0,3771$, respectivamente; $p<0,05$ ). Observou-se aumento de autoconfiança e segurança dos estudantes após aprendizagem simulada em manequim, que se mostrou ligeiramente mais valiosa para evolução desses quesitos em comparação à experiência clínica.
\end{abstract}

Descritores: Avaliação Educacional. Confiança. Manequins. Periodontia. Laboratórios Odontológicos.

\section{INTRODUÇÃO}

O Ensino Baseado em Simulação (EBS) evita colocar o paciente em risco e permite que o estudante reconheça suas eventuais falhas de conhecimento durante o processo de aprendizagem, além de permitir maior dedicação à 
técnica na prática simulada e que o estudante possa desenvolver melhores relações interpessoais e humanização no atendimento ao paciente ${ }^{1}$.

$\mathrm{O}$ uso de manequins simples (bonecos) foi introduzido na década de 1960 com o objetivo de melhorar o aprendizado, servindo como ferramenta de simulação entre as etapas aprendizado teórico e contato com pacientes, no ensino da medicina ${ }^{2}$. A simulação é uma metodologia ativa e racional para treinamento de habilidades básicas, que utiliza o ensino baseado em tarefas num ambiente protegido, seguro e controlado para reproduzir situações reais. Tal metodologia permite a repetida execução de uma tarefa, com pareceres imediatos, adequados e sistematizados acerca do que se pratica. Uma gama de objetos ou representações compõe esse sistema de ensino e aprendizado como manequins inertes, modelos anatômicos, realidade virtual, atores ou até a simulação de um ambiente completo $^{3}$.

Alguns estudantes se sentem insuficientemente preparados para sua primeira atuação clínica. Um estudo mostrou que esse sentimento nem sempre está ligado verdadeiramente à capacidade do estudante, mas sim com sua confiança ${ }^{4}$. Após aprendizagem simulada houve relatos de aumento de confiança e maior familiaridade com o ambiente ${ }^{4}$, além de pacientes terem classificado os estudantes que praticaram a simulação previamente como mais calmos e confiantes no momento do atendimento ${ }^{5}$. Contudo, apesar dessas vantagens, o EBS traz desafios aos docentes, que se deparam com estudantes em diferentes níveis de conhecimento teórico e desempenho, e precisam se adequar a cada um deles para um melhor aproveitamento da atividade prática simulada ${ }^{3}$. Adicionalmente, docentes entrevistados relataram baixo interesse de alguns estudantes para com a atividade por compreenderem a ferramenta utilizada apenas como um "boneco", encarando a situação com pouca seriedade ${ }^{3}$. Em específico para a área de
Odontologia, uma limitação descrita na literatura foi a carência de detalhes de um manequim ao reproduzir a mucosa oral ${ }^{5}$.

O objetivo deste estudo foi avaliar a evolução da autoconfiança e segurança de estudantes com acesso ao EBS no componente curricular de Periodontia I do curso de odontologia da Universidade Federal do Paraná (UFPR) no decorrer de um semestre.

\section{METODOLOGIA}

O estudo foi aprovado pelo Comitê de Ética em Pesquisa UFPR. O convite e todas as etapas de interação com os estudantes, como a aplicação do Termo de Consentimento Livre e Esclarecido e dos questionários, foram realizados pelos pesquisadores colaboradores e sem a presença do pesquisador responsável (que também é professor da Disciplina), a fim de assegurar a saudável relação professor-aluno, evitando situações de vulnerabilidade dos indivíduos e reduzida autonomia ou constrangimento. Da mesma maneira, todo o material obtido pelos pesquisadores colaboradores foi codificado, não havendo qualquer risco de retaliação por parte do docente aos estudantes não participantes ou constrangimentos quanto à autonomia individual.

O componente curricular de Periodontia I foi selecionado para execução desta metodologia pelo seu caráter teórico-prático, com frequência semanal e ambiente de ensino organizado, sendo o primeiro momento em que alunos deste curso têm contato com essa área de estudo. As três primeiras aulas são teóricas, seguidas de seis aulas para treinamento de raspagem e alisamento radicular em manequim, com outras quatro aulas na clínica para atendimento a pacientes.

Foram convidados para este estudo observacional longitudinal prospectivo todos os trinta e sete alunos matriculados na disciplina de 
Periodontia I da UFPR no primeiro semestre de 2018, de ambos os sexos, e com faixa etária variável, portanto, selecionados por conveniência. Após autorização e assinatura do Termo de Consentimento Livre e Esclarecido, um questionário foi aplicado aos estudantes em três momentos do período: 1) no início do semestre, antes de se iniciarem as atividades práticas; 2) dois meses depois, após a fase laboratorial; e 3 ) três meses depois da primeira aplicação, após a fase clínica. Foram consideradas a autoconfiança e segurança dos estudantes em realizar procedimentos clínicos em periodontia e raspagem e alisamento radicular, bem como seleção de instrumentos e instrução de higiene.

O questionário, composto por oito perguntas, foi elaborado pelos autores para responder à questão central da pesquisa (segurança e autoconfiança). A autoconfiança foi definida como confiança em si mesmo, ou seja, credibilidade ou conceito positivo que se tem a respeito de alguém ou de algo; crédito, segurança; crença de que algo é de qualidade superior e não falhará. Estabeleceu-se como segurança condição marcada por uma sensação de paz e tranquilidade, livre de riscos; força ou firmeza nos movimentos; confiança em si mesmo. ${ }^{6}$ Tais conceitos foram avaliados separadamente, já que o primeiro tem aspecto subjetivo de autoavaliação e o segundo representa a avaliação que o indivíduo tem sobre suas ações durante os procedimentos clínicos e laboratoriais. As perguntas realizadas constam do quadro 1.

Quadro1. Questionário utilizado para avaliar segurança e autoconfiança

- Qual seu nível de autoconfiança em realizar procedimentos clínicos em periodontia?

- Qual seu nível de autoconfiança em realizar raspagem e alisamento radicular?

- Qual seu nível de autoconfiança em selecionar instrumentais para raspagem alisamento radicular?

- Qual seu nível de autoconfiança em instruir um paciente quanto a higiene oral?

- Qual seu nível de segurança em realizar procedimentos clínicos em periodontia?

- Qual seu nível de segurança em realizar raspagem e alisamento radicular?

- Qual seu nível de segurança em selecionar instrumentais para raspagem alisamento radicular?

- Qual seu nível de segurança em instruir um paciente quanto a higiene oral?

Cada questão deveria ser respondida pelo estudante utilizando escala visual analógica (EVA), que consiste em uma linha de $10 \mathrm{~cm}$ ancorada por dois extremos, denominados "zero" e "altíssimo". O entrevistado é solicitado a fazer uma marca na linha que representa seu nível daquilo que se quer saber e a escala é pontuada medindo a distância da extremidade "zero" à marca do respondente; escala numérica de 101 pontos (NRS-101), na qual o entrevistado responde à questão escrevendo um número inteiro entre 0 e 100, sendo "0" representante de baixo nível e "100" de altíssimo nível de autoconfiança ou segurança; e escala verbal de 4 pontos (VRS-4), representada por uma lista de quatro adjetivos que descrevem os diferentes níveis, a saber: 0) nenhum; 1) baixo; 2) médio; 3 ) alto.

As aplicações tiveram duração de resposta média de 5 minutos, e foram realizadas no laboratório de periodontia, após as aulas práticas. As diferentes escalas utilizadas objetivaram a 
obtenção de diferentes variáveis (numérica discreta, numérica contínua e categórica ordinal).

As notas práticas foram conferidas por consenso entre os docentes da Disciplina, utilizando-se critérios representativos do rendimento acumulado durante o semestre. A cada aula prática (semanalmente) cada aluno foi avaliado em ficha individual contendo itens como "pontualidade", "material", "rendimento" e "outros". Ressalta-se que o os docentes da Disciplina estavam cegos aos resultados do estudo no momento do fechamento da nota prática.

Para os dados quantitativos, a distribuição normal foi avaliada utilizando o teste de Kolmogorov-Smirnov. Devido à distribuição não normal, foi aplicado o teste não paramétrico de Friedman com pós-teste de Dunn para comparações entre as aplicações inicial, após a fase laboratorial e após a fase clínica. Os mesmos testes foram aplicados para a variável categórica VRS-4. Para os quesitos autoconfiança e segurança, os dados foram tabulados agrupando- se as respostas às perguntas 1 a 4 e 5 a 8 , respectivamente. Tais quesitos foram correlacionados com a avaliação de desempenho laboratorial e clínico (média prática) dos estudantes por meio do teste de correlação de Spearman. Todos os testes foram realizados no software Prism 7 (GraphPad Software, La Jolla, Califórnia, USA) e o nível de significância foi estabelecido em $5 \%$.

\section{RESULTADOS}

Trinta e cinco indivíduos compuseram a amostra ao final do estudo. Dois foram excluídos da amostra inicial por deixar de comparecer a pelo menos uma das três aplicações dos questionários.

Quando os estudantes responderam usando a EVA, o tempo 2 foi estatisticamente diferente do tempo 1 para as perguntas 1 a $7(p<0,0001)$. Houve diferença significativa também entre os tempos 1 e 3 para todas as perguntas $(\mathrm{p}<0,0001)$ e entre os tempos 2 e 3 para as perguntas 1,3 e 5 $(\mathrm{p}<0,0001)$ (tabela 1).

Tabela 1. Média $\pm \mathrm{DP} /$ mediana ( $\Delta$ em relação ao tempo anterior) das respostas aferidas na Escala Visual Analógica (EVA) para cada pergunta (P) no início do semestre (Tempo 1), depois da fase laboratorial (Tempo 2) e depois da fase clínica (Tempo 3)

\begin{tabular}{ccccc}
\hline & Tempo 1 & Tempo 2 & Tempo 3 & p \\
\hline P1 & $30,26 \pm 20,22 / 27,9_{\mathrm{A}}$ & $48,86 \pm 15,6 / 48,9(18,6)_{\mathrm{B}}$ & $66,73 \pm 17,0 / 69,4(17,8)_{\mathrm{C}}$ & $<0,0001$ \\
P2 & $23,49 \pm 22,35 / 17,2_{\mathrm{A}}$ & $50,79 \pm 16,5 / 48,9(27,3)_{\mathrm{B}}$ & $55,99 \pm 22,34 / 57,3(5,2)_{\mathrm{B}}$ & $<0,0001$ \\
P3 & $18,47 \pm 17,07 / 12,6_{\mathrm{A}}$ & $64,99 \pm 22,35 / 67(46,52)_{\mathrm{B}}$ & $83,73 \pm 14,61 / 87,8(18,7)_{\mathrm{C}}$ & $<0,0001$ \\
P4 & $70,68 \pm 16,23 / 68,8_{\mathrm{A}}$ & $82,43 \pm 12,31 / 85,1(11,7)_{\mathrm{B}}$ & $87,29 \pm 12,62 / 89,4(5,3)_{\mathrm{B}}$ & $<0,0001$ \\
P5 & $24,25 \pm 20,42 / 27,9_{\mathrm{A}}$ & $50,61 \pm 18,09 / 48,9(26,3)_{\mathrm{B}}$ & $68,99 \pm 17,86 / 69,6(18,3)_{\mathrm{C}}$ & $<0,0001$ \\
P6 & $20,65 \pm 19,06 / 15,9_{\mathrm{A}}$ & $52,05 \pm 19,96 / 54,4(31,4)_{\mathrm{B}}$ & $62,7 \pm 24,15 / 68,4(10,6)_{\mathrm{B}}$ & $<0,0001$ \\
P7 & $17,46 \pm 15,63 / 13,8_{\mathrm{A}}$ & $67,15 \pm 22,43 / 74,4(49,6)_{\mathrm{B}}$ & $83,65 \pm 14,77 / 88,4(16,5)_{\mathrm{B}}$ & $<0,0001$ \\
P8 & $68,19 \pm 21,81 / 70,9_{\mathrm{A}}$ & $81,76 \pm 14,5 / 87,2(13,5)_{\mathrm{AB}}$ & $86,97 \pm 12,87 / 90(5,2)_{\mathrm{B}}$ & $<0,0001$ \\
\hline
\end{tabular}

Letras diferentes em cada linha representam diferenças estatisticamente significativas (Friedman com pós teste de Dunn). 
As respostas na NRS-101 mostraram diferença significativa para o tempo $1 \mathrm{em}$ todas as perguntas quando comparado aos tempos $2 \mathrm{e}$
3. O tempo 3 foi estatisticamente superior ao tempo 2 nas perguntas $1,3,5$ e $7(\mathrm{p}<0,0001)$ (tabela 2).

Tabela 2. Média $\pm \mathrm{DP} /$ mediana ( $\Delta$ em relação ao tempo anterior) das respostas aferidas na Escala Numérica de 101 pontos (NRS-101) para cada pergunta (P) no início do semestre (Tempo 1), depois da fase laboratorial (Tempo 2) e depois da fase clínica (Tempo 3)

\begin{tabular}{ccccc}
\hline & Tempo 1 & Tempo 2 & Tempo 3 & p \\
\hline P1 & $34,91 \pm 22,68 / 30_{\mathrm{A}}$ & $57,77 \pm 18,04 / 60(22,8)_{\mathrm{B}}$ & $70,77 \pm 15,25 / 70(13)_{\mathrm{C}}$ & $<0,0001$ \\
P2 & $27,54 \pm 22,42 / 20_{\mathrm{A}}$ & $58,23 \pm 15,86 / 60(30,7)_{\mathrm{B}}$ & $60,43 \pm 19,83 / 60(2,2)_{\mathrm{B}}$ & $<0,0001$ \\
P3 & $21,31 \pm 18,68 / 15_{\mathrm{A}}$ & $69,17 \pm 18,59 / 70(47,8)_{\mathrm{B}}$ & $84,77 \pm 13,43 / 90(15,6)_{\mathrm{C}}$ & $<0,0001$ \\
P4 & $73,57 \pm 15,32 / 80_{\mathrm{A}}$ & $84,74 \pm 11,19 / 90(11,2)_{\mathrm{B}}$ & $89,03 \pm 10,21 / 90(4,3)_{\mathrm{B}}$ & $<0,0001$ \\
P5 & $27,71 \pm 20,16 / 25_{\mathrm{A}}$ & $55,97 \pm 18,94 / 60(28,2)_{\mathrm{B}}$ & $68,2 \pm 15,54 / 70(12,2)_{\mathrm{C}}$ & $<0,0001$ \\
P6 & $24,54 \pm 19,12 / 20_{\mathrm{A}}$ & $54,14 \pm 17,8 / 60(29,6)_{\mathrm{B}}$ & $61,94 \pm 21,6 / 70(7,8)_{\mathrm{B}}$ & $<0,0001$ \\
P7 & $21,29 \pm 18,04 / 20_{\mathrm{A}}$ & $68,34 \pm 20,43 / 70(47,1)_{\mathrm{B}}$ & $84,97 \pm 12,83 / 90(16,6)_{\mathrm{C}}$ & $<0,0001$ \\
P8 & $70,97 \pm 19,8 / 70_{\mathrm{A}}$ & $84,66 \pm 11,97 / 90(13,7)_{\mathrm{B}}$ & $88,86 \pm 9,783 / 90(4,2)_{\mathrm{B}}$ & $<0,0001$ \\
\hline
\end{tabular}

A partir da VRS-4 foi possível observar que o tempo 1 foi inferior aos tempos 2 e 3 nas perguntas 1 a 3 e 5 a 7 (p<0,0001). Os tempos 2 e 3 não foram estatisticamente diferentes entre si em nenhuma das perguntas respondidas nessa escala. As perguntas 4 e 8 não demonstraram diferença entre as três aplicações ( $\mathrm{p}=0,0124 ; \mathrm{p}=0,0044)$ (figura 1).

Tanto na EVA quanto na NRS-101 a primeira, segunda e terceira aplicações foram significativamente diferentes entre si para autoconfiança e segurança, com elevação desses critérios no decorrer do semestre $(\mathrm{p}<0,0001)$ (figura 2).

A correlação entre a Média Prática (MP) dos estudantes e a autoconfiança para as escalas EVA e NRS-101 mostrou-se estatisticamente significativa (EVA: $r=0,3352 ; p=0,0490$; NRS-101: $r=0,3848$; $\mathrm{p}=0,0189$ ). A correlação entre MP e segurança na escala NRS-101 também se mostrou estatisticamente significante $(r=0,3771 ; p=0,0256)$. Não houve correlação estatisticamente significante entre MP e segurança quando a escala EVA foi utilizada $(r=0,266 ; p=0,1225)$ (figura 3 ).

\section{DISCUSSÃO}

Este estudo foi realizado com intuito de avaliar os efeitos de uma metodologia de ensino baseada em simulação na autoconfiança e segurança de estudantes de odontologia. Os achados deste trabalho mostraram que houve aumento da autoconfiança e segurança no decorrer do período, e correlação entre a autoconfiança e segurança (exceto em EVA) e o desempenho prático. No geral, os estudantes relataram estar mais autoconfiantes e seguros no final do período quando comparado ao primeiro dia de aula. Essa evolução foi notada principalmente entre a primeira e segunda aplicações dos questionários, ou seja, após a fase laboratorial simulada em manequim, que foi ligeiramente mais importante do que a experiência clínica para o aumento da autoconfiança e segurança dos estudantes.

O presente estudo encontrou aumento de segurança e autoconfiança dos estudantes de odon- 

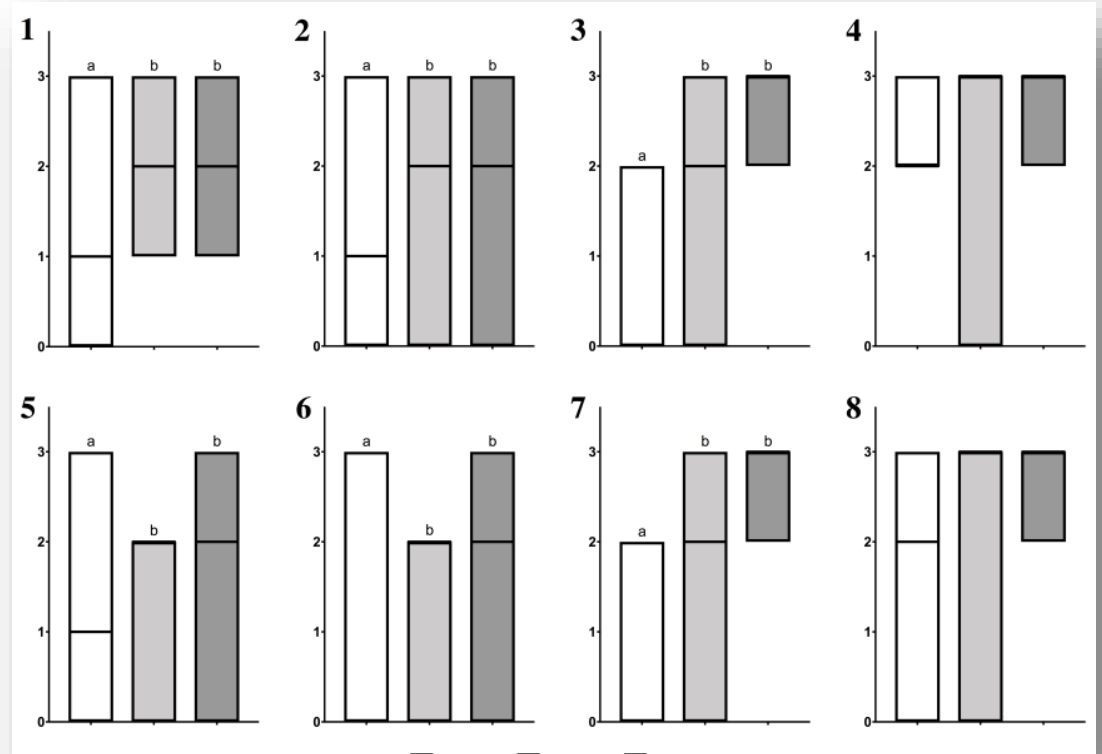

$\square$ Tempo $1 \square$ Tempo $2 \square$ Tempo 3

Figura 1. Medianas, máximas e mínimas das respostas aferidas na Escala verbal de 4 pontos (VRS-4) para cada pergunta (1 a 8) no início do semestre (Tempo 1), depois da fase laboratorial (Tempo 2) e depois da fase clínica (Tempo 3).cLetras diferentes em cada gráfico representam diferenças estatísticas significativas (Friedman; $\mathrm{p}<0,05$ )

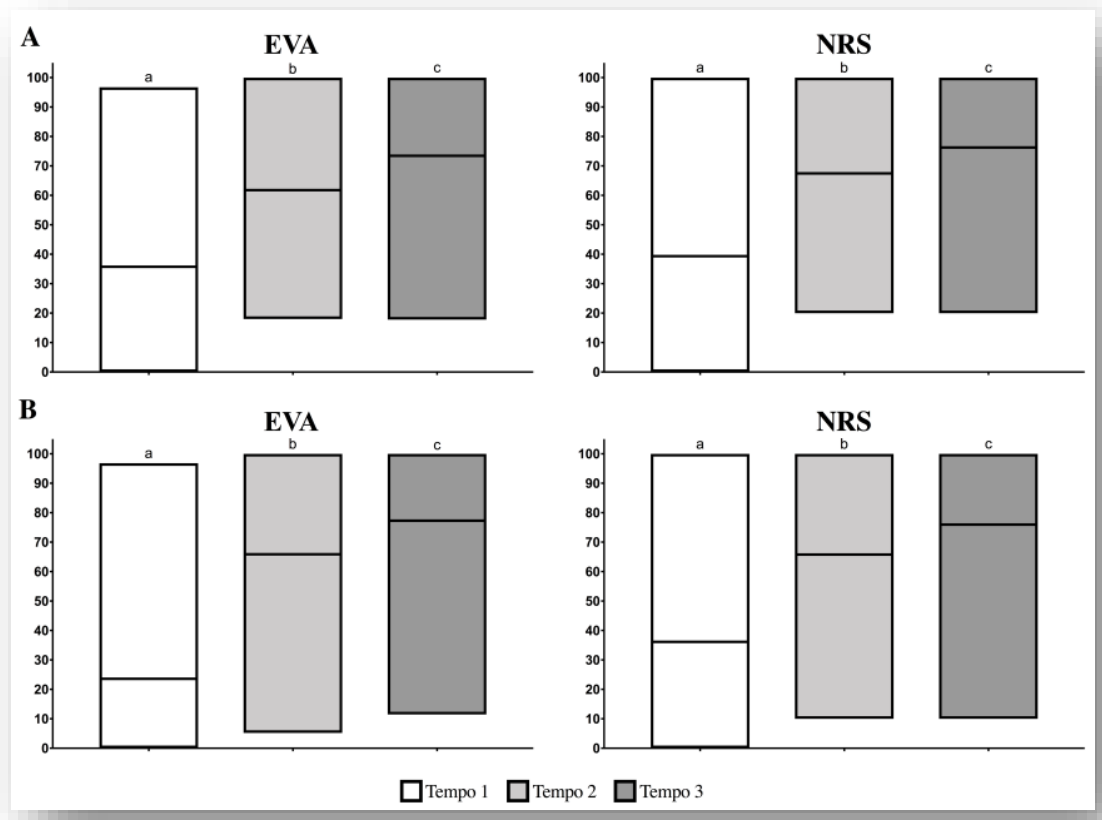

Figura 2 - Médias, máximas e mínimas das respostas aferidas na Escala Visual Analógica (EVA) e Escala Numérica de 101 pontos (NRS-101) para os quesitos Autoconfiança (A) e Segurança (B) no início do semestre (Tempo 1), depois da fase laboratorial (Tempo 2) e depois da fase clínica (Tempo 3). Letras diferentes em cada gráfico representam diferenças estatísticas significativas (Friedman; $\mathrm{p}<0,05$ ) 


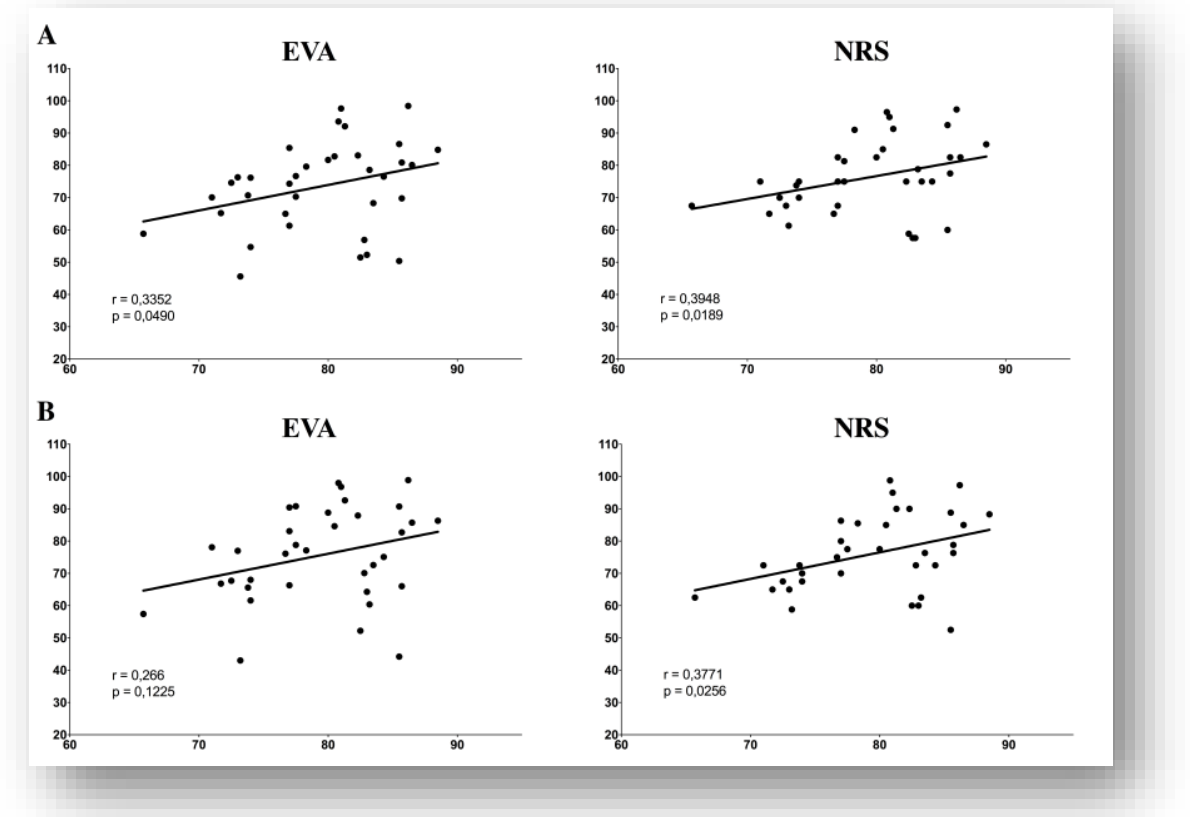

Figura 3. Correlação de Spearman entre Média Prática Final e Autoconfiança e Segurança aferidas na EVA e NRS-101 depois da fase clínica.

tologia na realização de procedimentos clínicos em periodontia, após execução de simulação. Esses achados estão de acordo com os observados em estudos anteriores, que reiteram a importância da simulação no contexto do aprendizado no ensino superior $^{4,7-10}$. Foi observado aumento estatisticamente significativo da autoconfiança e segurança avaliada pelas escalas de EVA e NRS-101 no decorrer da disciplina, ratificando desfechos encontrados na literatura, onde autores demonstraram ser a prática por meio de simulação um método eficaz de aprendizagem quando se trata de desenvolvimento de habilidades manuais e aumento da segurança na realização de procedimentos clínicos em situações reais ${ }^{4,8-10}$.

A experiência clínica mostrou efeito positivo, porém menos expressivo no aumento da autoconfiança e segurança quando comparada com o treinamento em manequim. Entre a aplicação inicial e após a fase laboratorial o aumento desses quesitos esteve entre 11,2 e 49,6 pontos, enquanto a evolução entre as aplicações após fase laboratorial e após fase clínica variou entre 2,2 e 18,7. Resultados semelhantes foram observados em outras pesquisas, que apontaram que a simulação não é alicerce para afirmar que os estudantes estão prontos para trabalhar com situações clínicas reais sem maiores intercorrências, porém é capaz de prover maior segurança e autonomia quando da exposição a um cenário verdadeiro ${ }^{2,3,11-14}$.

Assim como já exposto na literatura, notou-se correlação entre os níveis de autoconfiança e segurança dos estudantes e sua média prática final ${ }^{8}$. Entretanto, um outro estudo não encontrou diferença no desempenho clínico entre estudantes que receberam ou não o ensino simulado ${ }^{4}$.

Foram adotadas três escalas, que se apresentaram suficientes para medir um parâmetro pré-determinado em estudos de avaliação ${ }^{15}$. A EVA é sensível por apresentar inúmeras possibilidades de resposta, porém é 
difícil de ser aplicada e marcada. Com maior simplicidade e facilidade de aplicação, a VRS-4 tem apenas quatro opções de resposta com descrição, o que facilita o entendimento de quem responde, mas diminui sua sensibilidade. Já a NRS-101 é precisa, pois oferece âncoras que auxiliam o entrevistado no momento de definir a resposta (números inteiros entre 0 e 100), além de ser tão simples quanto a VRS-4 para aplicação e marcação ${ }^{16 .}$ As diferentes escalas utilizadas objetivaram a obtenção de diferentes variáveis (numérica discreta, numérica contínua e categórica ordinal), dessa forma abrangendo as possíveis discrepâncias entre respostas do mesmo indivíduo para a mesma pergunta.

Como limitações do presente estudo pode ser citado seu desenho observacional, o que dificulta a exploração das diferenças entre fase de simulação em manequim e fase clínica. Um desenho experimental no qual fosse possível separar grupos para ensino simulado e para experiência clínica poderia elucidar melhor as diferenças encontradas nos resultados.

\section{CONCLUSÃO}

Houve aumento na autoconfiança e segurança dos estudantes após aprendizagem simulada em manequim em periodontia, com correlação entre esses quesitos e o desempenho prático, além de essa metodologia de ensino ter se mostrado ligeiramente mais valiosa para evolução desses quesitos quando comparada à experiência clínica inicial.

\section{ABSTRACT \\ Evolution of students' self-confidence and trust after learning in periodontics manikins}

Simulation in health education is a tool that allows the student to learn in a protected and controlled environment, free from intercurrences and with instant feedback of the task performed. Simulation assists the instructor to evaluate the procedure performed and allows them to intervene at any moment, warning or instructing the student. This study aimed to evaluate the evolution of students' self-confidence and trust after simulated learning in a manikin during a curricular component of Periodontics and its correlation with practical performance. Questionnaires using visual analog scale (VAS), 101-point numeric rating scale (NRS-101) and 4point verbal rating scale (VRS-4) were applied to students enrolled in Periodontics I course at the Federal University of Paraná, Curitiba, Brazil, in the first term of 2018, in three different moments: 1) before the beginning of the practical activities; 2) after the laboratory phase; and 3) after the clinical phase. The data from the questionnaires were correlated with the final practical performance. Students reported being more selfconfident and secure at the end than the beginning of the term (Friedman; $p<0.05$ ). This evolution was noted mainly between the first and second questionnaire applications, after the laboratory phase with simulation. There was a correlation between self-confidence and trust measured in NRS-101 and the practical performance (Spearman; $r=0.3948$ and $r=0.3771$, respectively; $\mathrm{p}<0.05)$. Students' self-confidence and trust increased after simulated learning, which was slightly more valuable for the evolution of these aspects in comparison to the clinical experience.

Descriptors: Educational Measurement. Trust. Manikins. Periodontics. Laboratories, Dental.

\section{REFERÊNCIAS}

1. Dourado ASS, Giannella TR. Ensino baseado em simulação na formação continuada de médicos: análise das percepções de alunos e professores de um hospital do Rio de Janeiro. Rev Bras Educ Méd. 2014;38(4):460-9.

2. Mariani AW, Pêgo-Fernandes PM. Ensino médico: simulação e realidade virtual. Diagn Tratamento. 2012;17(2):47-8.

3. Iglesias AG, Pazin-Filho A. Emprego de simulações no ensino e na avaliação. Medicina (Ribeirão Preto). 2015;48(3):233-40.

4. Lee JS, Graham R, Bassiur JP, Lichtenthal 
RM. Evaluation of a local anesthesia simulation model with Dental students as novice clinicians. J Dent Educ. 2015; 79(12):1411-7.

5. Brand HS, Baart JA, Maas NE, Bachet I. Effect of a training model in local anesthesia teaching. J Dent Educ. 2010;74(8):876-9.

6. Michaelis Moderno Dicionário da Língua Portuguesa, 2015. [Acesso em 20 ago. 2018]. Disponível em: https://michaelis.uol.com.br/ moderno-portugues/busca/portugues-brasi leiro/

7. Costa RRO, Medeiros SM, Martins JCA, Menezes RMP. O uso da simulação no contexto da educação e formação em saúde e enfermagem: uma reflexão acadêmica. Espaç Saúde. 2015;16(1):59-65.

8. Volkweis MR, Oliveira AR, Faria FR, Wagner JCB, Gomes JP. Avaliação de uma técnica de exodontia em laboratório como instrumento de ensino - estudo piloto. Rev Cir Traumatol Buco-Maxilo-Fac. 2003; 3(1):31-6.

9. Tanalp J, Güven EP, Oktay I. Evaluation of dental students' perception and selfconfidence levels regarding endodontic treatment. Eur J Dent Educ. 2013;7(2):21824.

10. Celik Y, Ceylantekin Y. The evaluation of simulation market in nursing education and the determination of learning style of students. Int J Health Sci. 2017;11(1):13-8.

11. Arigbede A, Denloye O, Dosumu O. Use of simulators in operative dental education: experience in southern Nigeria. Afr Health Sci. 2015;15(1):269-77.

12. Heym R, Krause S, Hennessen T, Pitchika V, Ern C, Hickel R. A new model for training in periodontal examinations using manikins. J Dent Educ. 2016;80(12):1422-9.

13. Varga CRR, Almeida VC, Germano CMR, Melo DG, Chachá SGF, Souto BGA, et al. Relato de experiência: o uso de simulações no processo de ensino-aprendizagem em Medicina. Rev Bras Educ Med. 2009; 33(2):291-7.

14. Prasad S, Bansal N. Predoctoral Dental students' perceptions of dental implant training: effect of preclinical simulation and clinical experience. J Dent Educ. 2017;81(4): 395-403.

15. Hjermstad MJ, Fayers PM, Haugen DF, Caraceni A, Hanks GW, Loge JH, et al. Studies comparing numerical rating scales, verbal rating scales, and visual analogue scales for assessment of pain intensity in adults: a systematic literature review. J Pain Symptom Manage. 2011 41(6):1073-93.

16. Jensen MP, Karoly P, Braver S. The measurement of clinical pain intensity: a comparison of six methods. Pain. 1986 27(1):117-26.

\section{Correspondência para:}

João Paulo Steffens

e-mail: joao.steffens@ufpr.br

Departamento de Estomatologia

Universidade Federal do Paraná

Rua Pref. Lothário Meissner, 632

80210-170 Curitiba/PR 